\title{
Cabinets médicaux et médecine ambulatoire: que nous réserve le futur?
}

Ignazio Cassis

Conseiller national, vice-président de la FMH
Suite au rejet de la deuxième grande révision de la LAMal en décembre 2003, le Conseil fédéral a proposé des réformes séparées, pour éviter un front d'opposition trop large et tenter ainsi de faire un pas en avant. Non sans succès: en décembre 2007, nous avons approuvé au Parlement l'importante réforme du financement des hôpitaux, contre laquelle il n'y a pas eu de référendum. En juin 2008, nous avons accepté le nouveau régime de financement des soins de longue durée, qui résout le vieux problème du paiement des soins médicaux à domicile et dans les maisons de retraite. Restent maintenant sur la table trois paquets de révisions de la médecine ambulatoire, appelés respectivement «managed care» [04.062], "participation aux coûts» [04.034] et "liberté de contracter» [04.032]. Tous trois ont déjà été débattus au Conseil des Etats et sont maintenant examinés en détail par la commission de santé et de sécurité sociale du Conseil national.

\section{Après la médecine hospitalière, le moment est venu de réformer la médecine ambulatoire, qui a été jusque là l'objet d'interventions sporadiques dictées par \\ l'urgence}

Le blocage de l'accès à la pratique privée a induit de nombreux effets indésirables durant ces huit dernières années:

1. Dans les régions périphériques, il est toujours plus difficile de trouver des médecins disposés à reprendre l'activité d'un médecin de famille partant à la retraite; des zones apparaissent où la couverture médicale est insuffisante.

2. L'activité ambulatoire dans les hôpitaux a beaucoup augmenté, puisque ces derniers pouvaient contourner le blocage. Généralement plus coûteuse, cette activité a fortement alourdi la facture des soins.

3. La diffusion de modèles de type «managed care» - bien que réclamée à l'unisson par tous les acteurs - a été freinée par l'impossibilité, pour les médecins intéressés, d'obtenir l'autorisation cantonale nécessaire, même pour ces modèles.

4. Les nouveaux modèles de travail tenant compte des besoins des femmes-médecins mères de famille (cogestion à temps partiel d'un cabinet) ne sont en général pas autorisés.

5. L'application de cette règle fédérale est très hétérogène. D'un côté, cette hétérogénéité est simplement l'expression de notre fédéralisme, mais d'un autre, elle crée une grande insécurité juridique, malgré le fait que les professions médicales sont réglementées depuis 1877 par le droit fédéral.

Aussi était-il temps, après deux prorogations, de changer de registre. C'est pourquoi, avec nos collègues PDC Thérèse Meyer (FR) et Reto Wehrli (SZ) - et avec le soutien du PS et des Verts - nous avons présenté à la commission du Conseil national un projet destiné à remplacer la règle actuelle. Il s'agissait d'une proposition élaborée conjointement avec la FMH et la Conférence des directeurs cantonaux de la santé (CDS), que nous avions discutée et perfectionnée avec l'Office fédéral de la santé publique (OFSP) afin qu'elle ait le soutien du conseiller fédéral Couchepin. Nous y proposions des solutions concrètes aux problèmes rencontrés ces dernières années, y avions aussi inclus les pharmaciens, et étions d'accord d'en limiter la durée à 3 à 4 ans, le temps d'achever la réforme annoncée de la médecine ambulatoire. Mais nous avons perdu: par 13 voix contre 11, la commis- année - des accords bilatéraux Suisse-UE. Il devait s'agir d'une mesure d'urgence limitée à trois ans (jusqu'en 2005), destinée à déboucher sur une réforme globale de la médecine ambulatoire (cf. les trois paquets précités). Or, le Parlement n'a pas réussi en sept ans à trouver un nouveau plan global. Il est ainsi probable que ce blocage sera prolongé jusqu'à fin 2011, créant un préjudice important aux jeunes médecins désireux d'entreprendre une activité en cabinet. C'est ce qu'a décidé notre commission de la santé le 16 janvier. 
sion a préféré simplement prolonger encore le blocage, même si elle l'a fait seulement pour les spécialistes. Pourquoi en est-on arrivé là? La raison principale est simple: on aurait donné trop de pouvoir aux cantons, qui auraient pu planifier l'offre et réguler le marché sans assumer de responsabilité financière, vu que l'activité médicale ambulatoire reste entièrement payée par les caisses-maladie. Le principe «qui paie commande et qui commande paie» aurait été violé. Il y avait

\section{Cependant, le plus important se trouve dans la suite de l'exercice: quel type de médecine ambulatoire voulons-nous en Suisse? Et quelle facture sommes-nous prêts à payer?}

ture sommes-nous prêts à payer? Ce sont là les deux questions primordiales auxquelles il faudra répondre avant d'entrer dans les détails des trois projets du Conseil fédéral. Le $1^{\text {er }}$ juin 2008, les citoyens ont rejeté en votation populaire un renforcement du rôle des caisses-maladie et confirmé qu'ils voulaient pouvoir choisir entre différentes offres et différents médecins. Comment concilier une telle liberté avec le contrôle des coûts? Que devons-nous payer solidairement et que faut-il laisser à la charge de chacun? Voulons-nous des médecins professionnels indépendants ou toujours plus de fonctionnaires d'Etat? Plus de questions que de réponses. Aucun Etat n'a réussi à trouver une solution heureuse à tous ces problèmes. Même en Suisse, la solution sera forcément imparfaite, tant sont grandes les divergences d'intérêts. Une solution doit pourtant être trouvée pendant les deux prochaines années!

P.S. Les lecteurs intéressés ont la possibilité de connaître l'état des travaux en consultant les pages Web du Parlement. Copiez le lien: www. parlament.ch/F/dokumentation/do-dossiersaz/Pages/do-kvg2.aspx ou les codes des modèles ci-dessus (entre accolades) dans le champ «Recherche» du site www.parlement.ch. Bon surf! aussi la peur - à mon avis peu justifiée - d'une étatisation de la médecine ambulatoire: une solution inacceptable par la majorité, même de façon temporaire. Et nous voilà ainsi repartis pour une troisième prolongation du blocage de l'accès à la pratique privée.

Cependant, le plus important se trouve dans la suite de l'exercice: quel type de médecine ambulatoire voulons-nous en Suisse? Et quelle fac- 